\title{
FROM THE DESK OF EDITOR IN CHIEF
}

\section{From the Desk of Editor in Chief}

Editorial on "Adverse Effects of Tobacco on Pregnancy Outcome" showed Smoking during pregnancy can lead to a plethora of health risks to both the mother and the fetus. Women who smoke during pregnancy are about twice as likely to experience additional pregnancy complications. Tobacco is a preventable cause of adverse pregnancy outcome. Intervention to quit tobacco includes behavior therapy and pharmacotherapy.

Original Article on "Association of B-Type Natriureteric Peptide Levels With Estimated Glomerular Filtration Rate in Different Stages of Chronic Kidney Disease And Relevant Echocardiographic Changes" showed compelling evidence of the association of renal function and BNP, and emphasized that a single BNP measurement must be evaluated in conjunction with the entire clinical presentation, particularly the level of renal failure.

Article on "Comparative Study of Level of Serum Uric Acid in Type 2 Diabetes Mellitus Associated with Hypertension" showed that there were significant differences in mean of age, duration of DM, exercise time, waist hip ratio, systolic blood pressure and diastolic blood pressure within case and control.

Original article on "In Hospital outcome of Prediction of Peripheral Arterial disease In Diabetic Tobacco User Patients" showed that the awareness and implementation of $\mathrm{ABI}$ in general clinical practice is poor. A simple, inexpensive test like $\mathrm{ABI}$ can improve the diagnosis of PAD in clinical practice and thus help in preventing $\mathrm{CAD}$ and consequent death by a range of medical therapies.

Article on "Comparison of Left Radial versus Femoral Approaches for Coronary Procedures in Patients with Previous Coronary Artery Bypass Grafts" showed Contrast volume in between two groups was pretty similar and the incidence of other secondary endpoints was also not statistically significant.

Article on "Estimation of Serum Magnesium in Bangladeshi Gestational Diabetic Mother" revealed estimation of serum magnesium level should be incorporated in every GDM cases for prevention of complications

Article on "a prospective observational study on treatment evaluation in patients newly diagnosed with squamous cell carcinoma of head and neck in Bangladesh" revealed that squamous cell carcinoma of head and neck region are mostly presented in advanced stage in Bangladesh and the majority of the patients are treated with combination chemotherapeutic regimens. It emphasizes the need of early detection of these cancer.

Article on "Ejection Fraction < 35\% - Anaesthetic Experience of 236 Cases: A Retrospective Study" showed that Preoperative

AKMMC J 2018; 9(2) : 158 patient optimization, intraoperative haemodynamic stability and postoperative care have contributed to the success of very low ejection fraction patients in stay hospital.

Article On "Outcome of external dacryocysto-rhinostomy (DCR) operation without silicone tube intubation" re emphasized that Conventional DCR is the most common oculoplastic surgery performed for managing epiphora due to nasolacrimal duct obstruction.

Article on "Clinico-pathological profile and management outcome of Fournier's gangrene in a tertiary care hospital" concluded that the diagnosis and treatment of Fournier's gangrene is enigmatic and challenging to the physician. Urgent surgical debridement along with other appropriate supportive measures can reduce morbidity and mortality.

Article on "Comparative study of oral clonidine versus gabapentin as premedication for anxiolysis, sedation and attenuation of pressor response to laryngoscopy and tracheal intubation" showed that Oral clonidine provided good attenuation of hemodynamic response to laryngoscopy and intubation as compared with oral gabapentin. Also clonidine is better agent as anxiolytic and sedative than gabapentin.

Article on "Red cell distribution width (RDW) and $\mathrm{Hb} \%$ in the detection of iron deficiency anemia in pregnant women" revealed that Latent iron deficiency without other complicating disease could be screened out early by increased RDW when $\mathrm{Hb} \%$ was normal.

Review article on "Blood Component Therapy" showed that cellular components include packed red blood cells (PRBC), washed PRBC, leukoreduced PRBC and pooled or aphaeresis platelets. Plasma products such as FFP or cryoprecipitate, ant hemophilic factor (CRYO). The transfusion of red blood cells (RBCs), platelets, fresh-frozen plasma (FFP), and cryoprecipitate has the potential of improving clinical outcomes in perioperative and peripartum settings.

Case report on "Fordyce's Disease treated with Pimacrolimus" reports a rare case report and highlighted its cutaneous findings, histopathology and treatments outcomes.

Case report on "Crohn's Disease and Diagnostic Difficulty" reports diagnostic difficulties and atypical clinical presentation of Crohn's disesases emphasizes the early diagnosis \& installation of treatment.

I sincerely hope that this issue will be an informative one and interesting to the readers.

Prof. Dr. Md. Tahminur Rahman

MBBS, M. Phil, PhD,

Editor in Chief, AKMMC Journal 